\title{
Adjacent Tissue Transfer or Rearrangement
}

National Cancer Institute

\section{Source}

National Cancer Institute. Adjacent Tissue Transfer or Rearrangement. NCI Thesaurus. Code C160576.

A reconstructive procedure that relocates a flap of healthy skin from a donor site to an adjacent laceration, scar, or other discontinuity. A portion of the flap is left intact to supply blood to the grafted area. Adjacent tissue transfer/rearrangement (ATT/R) may be for repair of traumatic skin wounds, lesion excision, or rearrang ement/reconstruction of tissues. 